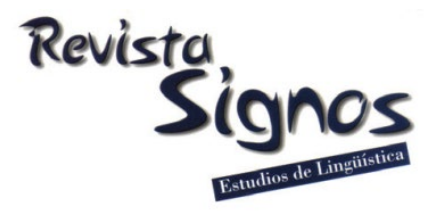

\title{
Semántica y productividad de la clase nominal en mocoví
}

\section{Semantics and productivity of the nominal class in Mocovi}

\section{Cintia Carrió}

INSTITUTO DE HUMANIDADES Y CiENCIAS SOCIALES DEL LITORAL CONICET/UNL

ARGENTINA

cintiacarrio@conicet.gov.ar

\author{
Valentina Jara \\ UNIVERSIDAD NACIONAL DEL LITORAL \\ ARGENTINA \\ jara.valentina26@gmail.com
}

María Inés

Rabasedas

INSTITUTO DE HUMANIDADES Y Ciencias Sociales del LitoraL CONICET/UNI ARGENTINA

ines.rabasedas@gmail.com

Recibido: 30-VI-2018 / Aceptado: 12-XII-2018

DOI: $10.4067 /$ S0718-09342019000200639

\section{Resumen}

El objetivo del presente trabajo es delimitar la categoría 'nombre' en la gramática del mocoví. Interesa discutir la semántica nominal, atendiendo al fenómeno de la delimitación de clases. Para ello nos centramos en las particularidades morfológicas de los nombres y las nominalizaciones, y proponemos un análisis que atiende al comportamiento de esta categoría en interfaz con la sintaxis y la morfología. En cuanto a la metodología empleada, se analizaron datos de las variedades chaqueña y santafesina. Se consideraron, además, los datos de la bibliografía sistemática básica disponible sobre la lengua. Esta muestra se completó con textos libres y orales que son producto de elicitaciones directas y contextualizadas mediante la proyección de imágenes. Si bien se observó que la categoría 'nombre' comparte características comunes con los adjetivos y verbos de la lengua, se reconocieron propiedades morfosintácticas que permiten delimitarla como una categoría diferenciada. Se pudo reconocer, también, que el mocoví parece disponer de rasgos puramente sintácticos que posibilitan la generación productiva de nombres complejos. A su vez, se identificaron rasgos semánticos asociados a diferentes sufijos nominalizadores, tales como: locación, semántica vegetal, eventividad, resultado, instrumento y agentividad.

Palabras Clave: Nombre, nominalizaciones, morfosintaxis, lenguas aborígenes, Gran Chaco. 


\begin{abstract}
The aim of this paper is to delimit the category nombre within Mocoví grammar. It is our interest to discuss nominal semantics, based on grammatical class delimitation. To do so, we focus on the morphological features of the names and on nominalizations. We also propose an analysis on the behavior of this category in interface with syntax and morphology. Regarding methodology, data taken from Mocoví branches from Chaco and Santa Fe were analyzed. Available basic systematic bibliography about the language was also taken into account. This sample was completed with free and oral texts, gathered by direct and contextualized elicitation, by means of image projection. Although it was noticed that the category 'name' shares common features with adjectives and verbs of the language, singular morphosyntactic properties were recognized as to label it as a differentiated category. It was also possible to be recognized that Mocoví possesses purely syntactic features, which allow the productive creation of complex names. In addition to this, semantic features associated to different nominalization suffixes such as location, vegetal semantics, eventiveness, result, instrument and agency were identified.
\end{abstract}

Key Words: Noun, nominalizations, morphosyntax, native languages, Gran Chaco.

\title{
INTRODUCCIÓN1
}

Al pueblo mocoví le pertenece la lengua de su mismo nombre que se habla sólo en territorio argentino. Esta es una lengua de la familia Guaycurú en la que pueden diferenciarse dos variedades, localizadas en dos regiones geográficas más o menos específicas, a saber, una variedad chaqueña ${ }^{2}$ (o de la macro-región chaqueña), en la que se evidencia mayor contacto con otras lenguas originarias (toba y wichi, por ejemplo), y una variedad santafesina, con menor vitalidad que la anterior. Estas dos variedades se diferencian fundamentalmente por rasgos fonológicos.

En lo que respecta específicamente a la problemática central de este artículo, se reconocen, como antecedentes para el estudio de los nombres y las nominalizaciones en mocoví, los trabajos de Grondona (1998), Gualdieri (1998) y Carrió (2009). En Grondona (1998) se distinguen dos tipos de raíces nominales: libres y ligadas. Se propone que las raíces ligadas (1) no tienen independencia sintáctica y deben estar precedidas por un proclítico posesivo o uno absolutivo (por ejemplo, términos de parentesco, partes del cuerpo y objetos hechos por el hombre). En cambio, las raíces libres (2) son las que pueden ocurrir con independencia sintáctica, sin ningún marcador posesivo o absolutivo (generalmente, animales, elementos de la naturaleza, préstamos y referencias a entidades humanas como hombre o mujer).
(1) s-ogoren-e
ke-na
nakalpe
na $\quad i-\beta o$
1-tener.lástima-PL OBL-DET niño.PL
'Les tengo lástima a los chicos de mi casa.'
DET 1POS-hogar
(Texto inédito 1) 
(2) kenaa i-noGon-ta-ni rahasa

CONJ 3-esconder-DUR-DIR sol

'...y cuando se escondía el sol...'

$(\mathrm{PIO} 1)^{3}$

Por otra parte, Gualdieri $(1998,2003)$ analiza distintos procesos morfológicos que permiten derivar nombres. En sus escritos distingue una serie de sufijos que derivan nombres a partir de bases verbales y una serie de sufijos que permiten derivar nombres de bases nominales. En Carrió (2009) se analizan con mayor detalle las nominalizaciones eventivas y resultativas; y en Carrió (2015a; 2015b) las nominalizaciones de instrumento.

En Carrió (2009) se estudian puntualmente las nominalizaciones que se generan por derivación de raíces nominales y verbales con morfemas nominalizadores particulares. En este sentido, se sostiene que hay morfemas nominalizadores que permiten generar lecturas eventiva y resultativa. El morfema -ek (masculino) (3) y -a (femenino) (4) generan nominalizaciones resultativas; mientras que, el morfema -aGak, eventivas (5); ambas nominalizaciones son posibles a partir de raíces verbales sujetas a ciertos condicionamientos.

(3) ono-oki i-ußiyi-ek

uno-DIM 1POS-fijar-RES.M

'un solo pescado'

(4) so jale ve l-ogon-a

DET hombre EX 3POS-cazar-RES.F

'Aquel hombre tiene su presa.'

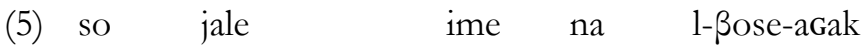

DET hombre ADV DET 3POS-cocinar-EV

'Aquel hombre terminó de cocinar.'

Estos casos se asumen como derivados, dado que las nominalizaciones presentan más material morfológico que los verbos correspondientes. Concretamente, se analizan algunas características de las nominalizaciones eventivas, esto es: (i) presentan flexión pronominal obligatoria correferente con el agente del verbo base (6); (ii) hacen posible referir directamente al evento o proceso; y parecen tener restringida la posición de sujeto oracional; (iii) no pueden saturar la posición de argumento interno de verbos transitivos que suponen un complemento con el rol temático tema, y no pueden ser complementos de construcciones existenciales dado que no presentan usos predicativos (7). 
(6) nayi ime ii-egon-agak na i-po si-egon-Gan-tak kaßit

ADV ADV 1POS-tejer-EV DET 1POS-poncho 1-tejer-ANTP-PROG ADV

'Hoy terminé de tejer mi poncho que estaba tejiendo ayer.'

$\begin{array}{llll}(7) * \quad \text { xuan } \quad \text { i-okat } \int a a & \text { so } & \text { i-owen-aGak } \\ \text { Juan } & \text { 3-robar } & \text { DET } & \text { 1POS-trabajar-EV } \\ \text { 'Juan robó mi trabajo.' } & & \end{array}$

Con respecto a las nominalizaciones resultativas, se sostiene que: (i) pueden pluralizarse (8); (ii) si están acompañadas de otro nominal, se establece concordancia de género y número entre el resultativo y el nominal-objeto tema (excepto cuando hay presencia de cuantificador) (8); y, (iii) el posesivo se comporta como complemento, ya que está ligado a una posición en la estructura léxico conceptual del nombre. En este sentido, Carrió (2009) postula que las nominalizaciones resultativas heredan la estructura argumental de los verbos de los que derivan (9).

(8) tfalo i-ogon-a

lowani

kee-da

la'tewe

cuant 1pos-cazar-res.pl

pato.sg

obl-det

río

'Cacé muchos patos en el río.' (Lit.: 'muchas mis.presas pato en el río.')

(9) $l_{i}-e r-e k$

xuan

3pos-escribir-res

Juan

‘el libro de juan' [con interpretación agentiva: 'lo que Juan escribió']

Esto permite concluir que tanto las resultativas como las eventivas presentan estructura argumental y cuentan con un argumento externo agentivo. Se destaca, además, que el morfema -aGak focaliza la atención en el desarrollo del proceso mismo, nominalizando acciones y estados; mientras que el morfema -ek hace hincapié en el resultado de ese proceso derivando nombres que refieren a argumentos verbales. Entonces, las formas eventivas aceptan modificadores de manera, mientras que las resultativas habilitan modificadores de objeto. Además, las nominalizaciones resultativas implican, en su estructura interna, estructuras eventivas que conducen a un cambio de estado, suponen afectación. Esto explica la mala formación de los resultativos derivados de bases inergativas sin alternancia transitiva. Finalmente, se postula que las nominalizaciones resultativas seleccionan como base verbos con dos posiciones argumentales (o bases inergativas que presentan alternancia transitiva); presentan un argumento interno tema afectado cuya aparición junto al núcleo nominal es opcional, y presenta un argumento externo agentivo de realización obligatoria que se ensambla con la base mediante un prefijo posesivo correferente con el argumento externo del verbo base de la derivación.

En Carrió (2015b) se estudia la causatividad como un universal semántico translingüístico que se vale de distintos fenómenos. Se distinguen diferentes tipos de construcciones causativas: léxicas, morfológicas y analíticas; y se diferencia la 
causación directa (causa agentiva y causado afectado) de la indirecta (dos participantes agentivos y dos eventos). El estudio de la causación en estas distintas dimensiones permite analizar fenómenos como las nominalizaciones, en tanto éstas derivan de bases verbales. En este sentido, analiza la morfología nominal resultativa (a diferencia de la eventiva) como implicada en la semántica causativa, y también trabaja con nominalizaciones instrumentales. Carrió sostiene que las nominalizaciones instrumentales son nombres que se derivan de raíces verbales no-estativas mediante el sufijo -Gat para el género masculino y -qate para el femenino (-Gat-e) (cfr. 3.1.2.3.)

(10) ke-na lepe piPogon-aq-a

OBL-DET noche brujo-NMZ.AG-F INSTR

n-pio?-ta $\quad 1-\beta o$

IND-sucio-DUR 3POS-sangre

'Cuando llegó la noche, la "bruja" tiró al agua un hilo embadurnado con sangre.'

(11) 1-Plek-Gan-qate

3POS-barrer- ANTP-INSTR.F

'Su escoba (de él/ella)'

Retoma a Gualdieri (1998) para proponer que los instrumentos son instancias de iniciadores semánticos codificados frecuentemente por causantes (iniciadores típicos).

Por último aquí, en Carrió (2015a) se estudia la alternancia en la realización sintáctica de la estructura argumental, como indicador de la relación sistemática que existe entre el léxico y la sintaxis. Sostiene que la alternancia verbal comprende las distintas asociaciones que se pueden generar entre las funciones semánticas de los argumentos exigidos por el verbo y, además, sus funciones sintácticas. En este sentido, distingue cinco tipos de alternancias en el dominio verbal del mocoví: (i) alternancias causativas, entre las que está (a) la alternancia transitiva-causativa de cambio locativo (12) y (b) la alternancia incoativa-causativa (13); (ii) alternancia antipasiva (14); (iii) alternancia de tema implícito y alternancia resultativa (15); (iv) alternancia sujeto/adjunto entre las que se encuentra la (a) alternancia sujeto/instrumento (16) y (b) sujeto/causa (17); y, por último, la (v) alternancia oblicuo/aplicativo (18).

(12) a.

$$
\text { nua iat'al ve-sa-yenaa }
$$

eskalada

DET.PL padres EX-DUR-APL lugar Escalada

'Mis padres están en Escalada'.

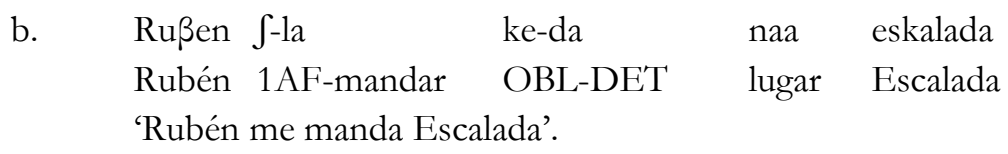



a. so l-ate ?e kijo so l-iale

DET 3POS-madre lavar DET 3POS-hijo.F

'La mamá lava a su hija.'

b. jim kijo- Gan-tak

PRO1 lavar-ANTP-PROG

'Yo estoy lavando.'

(14) s-par- Gan-o

1-cazar-ANT-PROS

'Voy a cazar.'
a. $\quad$ da $\quad$ i-filo-aßa
r-eßose-tak
nai

DET 1POS-casamiento-COM 3-cocinar-PROG pescado

1-'lege

tißiagar-aik

3POS-condimento

ser.ácido-ADJ

'Mi compañero está cocinando pescado condimentado con limón.'

b. da i-filo-aßa l-eßos-ek

DET 1POS-casamiento-COM 3-cocinar-RES

(nai l-'lege tißiagar-aik)

(pescado 3POS-condimento ser.ácido-ADJ)

'La comida que hizo mi compañero es pescado condimentado con limón.'
a. so ahlo
i-a $\int \mathrm{i} B \mathrm{Bi}-\mathrm{Gat}$
so nogot-oli
DET mujer 3-secar-CAU DET
nene-DIM.F

ke-na

n-a $\int i ß i a-q a t-G a n-G a t$

OBL-DET

POSIND-secar-?-ANTP-INSTR.M

'La mujer seca a la nenita con una toalla.'

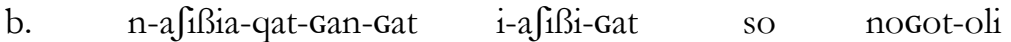

POSIND-secar-?-ANTP-INSTR.M 3-secar-CAU DET nene-DIM.F

'La toalla seca a la nenita.'
a. jim pa?e-Gat
na
BaGaiak ke-na l-avak rahasa

PRO1 caliente-CAU DET agua OBL-DET 3POS-rayo sol 'Yo calenté el agua con el reflejo del sol.'

b. ni l-avak rahasa pa?e-Gat na Bagaiak DET 3POS-rayo sol caliente-CAU DET agua

'El reflejo del sol calentó el agua.'
a. s-not-tak
(ke-na
nik)
1-saltar- PROG OBL-DET
soga
'Estoy saltando (con la soga)' 


$$
\begin{aligned}
& \text { b. xuan } r \text {-not-lek na tema' } \\
& \text { juan 3-saltar-APL DET pozo.(con agua) } \\
& \text { 'Juan saltó sobre el pozo con agua [al otro lado]' }
\end{aligned}
$$

Si bien se centra en el estudio de las alternancias verbales en mocoví, en (iii) se observa el fenómeno de las nominalizaciones resultativas. Dentro de este tipo de alternancia, se propone que las nominalizaciones resultativas heredan la estructura argumental de los verbos de los que derivan y se muestran las pruebas que sostienen esta afirmación.

Los sufijos que derivan nombres a partir de bases nominales no recibieron la misma atención que los casos anteriores. Al momento solo se dispone de una breve descripción (Gualdieri, 1998) en la que se listan seis sufijos de este tipo: -lad; -ki; -wa; le/-lek; -se/ -sek; -ik; -sat.

(19) kePe-jaga-lad

(Gualdieri, 1998: 165) comer-NMZ-CL 'mesa'

(20) n-qoin-Gan-aGa-ki (Gualdieri, 1998: 165) IND-apresar-ANTP-NMZ-CL 'trampa para cazar'

(21) pa?yen-aGa-wa (Gualdieri, 1998: 167) envidiar-NMZ-CL 'enemigo'

(22) kotaiol-ek

$$
\begin{array}{lll}
\text { rahan-sak } & \text { ke-da } & \text { piyim } \\
\text { 3-mirar-PROG } & \text { OBL-DET } & \text { cielo }
\end{array}
$$
dios-M

'Dios los está mirando desde el cielo'

(23) nete?e-se mañana-CL.F 'estrella matutina'

(24) nor-ik quebracho-CL 'quebracho blanco'

(25) t tawik-sat palmera-CL 'palmar'
(Gualdieri, 1998: 170)

(Gualdieri, 1998: 172)

(Gualdieri, 1998: 172)

La preocupación central aquí es delimitar la categoría 'nombre' en la gramática del mocoví. Interesa discutir la semántica nominal, atendiendo al fenómeno de la 
delimitación de clases. Para ello nos centramos en las particularidades morfológicas de los nombres y las nominalizaciones, y proponemos un análisis que atiende al comportamiento de esta categoría en interfaz con la sintaxis y la morfología.

Entre las cuestiones teóricas relevantes que este trabajo propone se encuentran las siguientes: (1) cómo está organizada la gramática de esta lengua particular; (2) cuáles son los rasgos disponibles para la generación de nominalizaciones; (3) cuáles son las restricciones que se le presentan; y, (4) cuál es su productividad.

Para atender a estos objetivos, describimos las características centrales de las nominalizaciones; revisamos los contextos morfológicos y sintácticos en las que aparecen, y las restricciones que se les imponen; analizamos especialmente las operaciones en las que participan y aquellas por las cuales atraviesan; y, describimos la semántica de cada caso. Consideramos que este trabajo abre diversas posibilidades para discutir y reanalizar esta categoría de alta productividad en esta lengua.

En el apartado 1. se detallan las consideraciones teóricas asumidas y las hipótesis esbozadas. En el apartado 2. se explicita el marco metodológico de este trabajo. En el apartado 3. describimos y discutimos los datos que nos permiten avanzar sobre las hipótesis de trabajo; se muestra la productividad de las construcciones nominales, sus rasgos morfológicos centrales, sus características y condicionamientos sintácticos, y se discute respecto de algunas barreras intercategoriales que a primera vista podrían parecer lábiles. Por último, en el apartado 4 exponemos las conclusiones.

\section{Marco teórico}

Las tesis doctorales de Gualdieri (1998), Grondona (1998) y Carrió (2009), junto con la tesis de maestría de Juárez (2013) y la tesina de licenciatura de Rabasedas (2016) son las sistematizaciones más controladas con las que se cuenta. Cabe aclarar que Gualdieri (1998), Grondona (1998) y Juárez (2013) analizan la variedad chaqueña de la lengua, mientras que los trabajos de Carrió y Rabasedas se enfocan en la variedad santafesina.

El nombre es una categoría de alta frecuencia en esta lengua ya que aparece involucrado en diferentes fenómenos, muchos de los cuales suelen estar asociados tradicionalmente, en las lenguas del mundo, a otras categorías. Entre los estudios previos sobre la lengua no se cuenta con análisis de alto alcance que hayan focalizado específicamente en el fenómeno de las nominalizaciones, tanto las que derivan de bases verbales como las que derivan de bases no verbales. No obstante esto, cabe destacar que la tesis de Gualdieri (1998) reconoce una serie de sufijos derivativos que permiten la generación de distintos tipos de nominalizaciones. Mientras que en Carrió (2009) se analizan con mayor detalle las nominalizaciones eventivas y resultativas; y en Carrió (2015a, 2015b) las nominalizaciones de instrumento. Sobre esta problemática avanza concretamente el trabajo de Jara (en desarrollo). Los nombres funcionan en la 
gramática como argumentos o núcleos de argumentos. En mocoví, también cumplen la función de predicados en construcciones ecuativas que no presentan cópula expresa. Esta característica, al margen de otras peculiaridades morfosintácticas, parece indicar que, en ciertas lenguas, la distinción 'universal' nombre/verbo no puede ser claramente establecida. Son miembros de la clase de los nombres aquellas palabras que cargan especificaciones, en la morfología y/o en la sintaxis, para número, género/clase y definitud. La categoría de Caso, típicamente atribuida a los nombres, no es marcada en ellos, sino en el núcleo (el verbo, para los Casos nucleares; el nombre poseído, en las construcciones genitivas).

A diferencia de los verbos, los nombres presentan, en mocoví, una distinción tripartita de número expresado morfológicamente: singular, dual/paucal y plural/colectivo. El género femenino/masculino es marcado morfosintácticamente por afijos de clase, por la concordancia en los clasificadores nominales y por sufijos exclusivamente de género. Las clases nominales son marcadas ya sea por sufijos, que expresan otras funciones además de clase, o por los ítems analizados como clasificadores nominales. Éstos también contribuyen en la especificación de definitud.

En esta propuesta, se consideran los aportes de la Morfología Distribuida (MD) (Halle \& Marantz, 1993; Embick \& Noyer, 2004; Bobaljik, 2015, entre otros) ya que desde este enfoque se piensa a las raíces como elementos que ingresan a la sintaxis sin especificación categorial, lo cual permite postular que éstas pueden combinarse con diferentes categorizadores en distintos contextos (Marantz, 1997, 2001). Así, la MD permite un análisis alternativo de los datos para avanzar en el estudio de los nombres y nominalizaciones (Arad, 2003; Harley, 2009).

Investigaciones previas han estudiado diversas problemáticas vinculadas con la gramática de esta lengua. Estas investigaciones permiten poder postular hipótesis descriptivas y explicativas respecto del funcionamiento de esta gramática particular. En la presente propuesta, se busca describir las particularidades morfológicas de los nombres y las nominalizaciones a partir de cómo se comportan en interfaz con la sintaxis y la morfología. En función de esto, se busca analizar los rasgos disponibles en el inventario de la gramática del mocoví para la generación de los nombres en la lengua.

Este análisis permitirá, por un lado, revisar los postulados y afirmaciones básicos sobre los nombres y las nominalizaciones en mocoví desarrollados en los trabajos previos sobre la lengua (ver Introducción); y, por otro, profundizar en la explicación de algunos fenómenos morfosintácticos de la formación de palabras en mocoví. Así se pretende definir algunas líneas de análisis a partir del trabajo de datos de la lengua mocoví, que implican reconsiderar la funcionalidad de las raíces no categorizadas. 
Se trabaja recuperando los supuestos de la $\mathrm{MD}$, según la cual, existe un único sistema generativo para formar palabras. Esta postura contrasta con las teorías lexicalistas, según las cuales existen dos componentes diferentes en la gramática: uno para crear palabras (el léxico) y otro para crear frases y oraciones (la sintaxis). Al considerar que las palabras también son objetos complejos, cuya formación obedece a las reglas de la sintaxis, la MD considera que la gramática de las lenguas no cuentan con un componente 'léxico', pues tanto las palabras como las frases y oraciones se forman por las mismas operaciones: 'ensamble' y 'movimiento'. Así, se postula la existencia de una interfaz transparente entre sintaxis y morfología, siendo el mismo componente (la sintaxis) el que deriva todos los objetos complejos.

Desde esta teoría, se asume que la sintaxis manipula dos tipos de unidades: los morfemas funcionales, por un lado, y las raíces, por el otro. En el primer caso, estos elementos están compuestos por rasgos symsem (sintáctico-semántico), pero no poseen contenido fonológico, ya que están sujetos a inserción tardía. Una particularidad de la teoría es que incluye como morfemas funcionales a los categorizadores, esto es, a aquellos núcleos funcionales que proveen las categorías léxicas, tales como $n, v, a$. De esta forma, la distinción entre morfemas flexivos y derivativos pierde valor en la MD, ya que ambos tipos de morfemas se conciben como funcionales. Las raíces, por su parte, son miembros de la clase abierta del vocabulario, y se asumen como carentes de especificación categorial. En la MD se reconocen diferentes hipótesis en relación al contenido de las raíces, ya que en algunos casos se asume que presentan contenido fonológico y propiedades semánticas inherentes, denominadas 'significados-R' (Embick, 2015), mientras que en otros se considera que no poseen ninguno de estos contenidos sino que se encuentran identificadas mediante índices (Harley, 2014). En este trabajo, se asume la primera postura ya que permite explicar ciertas interacciones que ocurren entre las representaciones léxico-semánticas de las raíces y los categorizadores que las seleccionan.

Así, desde la MD se asume que una raíz ingresa a la sintaxis sin especificación categorial. En relación con este aspecto, se propone la denominada 'asunción de categorización', según la cual las raíces no pueden aparecer desnudas por lo que son categorizadas en estructuras sintácticas por núcleos funcionales definidores de categoría. Entonces, desde este enfoque, ciertas proyecciones funcionales son las encargadas de categorizar a las raíces. Las palabras tradicionalmente consideradas simples se asumen como estructuras complejas, en las que el morfema categorizador no contiene material fonológico.

\section{Marco metodológico}

Para esta investigación se consideran datos de las dos variedades de la lengua, las variedades chaqueña y santafesina; si bien predominan los correspondientes a esta última variedad. Se consideraron, además, los datos de la bibliografía sistemática básica 
disponible sobre la lengua, esto es, las reconstrucciones gramaticales de las tesis de Gualdieri (1998), Grondona (1998), Carrió (2009), Juárez (2013), Rabasedas (2016); los glosarios de Bucca (1979) y Buckwalter y Ruiz (2000) y el diccionario de Buckwalter y Buckwalter (2004).

Esta muestra se completó con una serie de textos libres orales y registros de campo constituidos por aproximadamente 4500 entradas sintagmáticas, producto de elicitaciones directas y contextualizadas mediante la proyección de imágenes, todo ello grabado en las comunidades de ialek lavá (Colonia Dolores, departamento San Justo), Tilala (en la localidad de Marcelino Escalada, departamento San Justo) La Thee Palma (Berna, departamento General Obligado) y Com Caiá (Barrio Comunal de Recreo, departamento La Capital) en periodos discontinuos desde el año 2007 hasta el año 2018 y en visitas esporádicas entre 2004 y 2006. Los datos incorporados a este trabajo se transcriben con el alfabeto fonético internacional dado que no hay consenso respecto de la escritura en mocoví. En las comunidades con las que se trabaja coexisten diferentes alfabetos e, incluso, en la mayoría de los casos no ha habido una discusión reflexiva (ya sea en términos lingüísticos, ya en términos políticos) respecto de la estandarización y/o algún tipo de consenso.

Cabe destacar que la recolección de los datos de primera mano estuvo a cargo de los autores de este trabajo. En lo que respecta específicamente a los textos libres, estos responden a diferentes géneros discursivos (cuento, poesía, consejo, relato de vida); en lo relacionado con la elicitación mediante imágenes, se aplicó un instrumento creado específicamente para la obtención de descripciones orientadas al campo semántico de la salud; los referentes (hablantes nativos de entre 50 y 70 años) fueron expuestos a una sucesión de imágenes que describían con diferente nivel de fluidez. Las micronarraciones obtenidas fueron transcriptas y controladas con más de un hablante.

En la sección siguiente se presentan y analizan los datos. Dicha presentación se organiza de la siguiente manera: en una primera instancia, se describe la estructura morfológica de los nombres y nominalizaciones de la lengua; en una segunda instancia, y en relación a la naturaleza categorial de los nombres en mocoví, se trabajan las semejanzas y diferencias morfosintácticas entre esta clase de palabra, por un lado, y los adjetivos y verbos de la lengua, por el otro.

\section{Datos y discusión}

Antes de presentar los datos que permiten la mostración y discusión del problema, cabe destacar algunas de las particularidades de la lengua observada, a fin de lograr una mejor comprensión de los fenómenos analizados.

La lengua mocoví presenta el orden no marcado AVO para las oraciones simples de dos argumentos y la alternancia SV y VS para el caso de las oraciones 
monoargumentales. Es una lengua que marca el núcleo; es flexiva, no admite raíces verbales libres y no presenta morfología temporal marcada en el verbo. La operación sintáctica de ensamble es altamente productiva, a través de ella se logran construcciones atributivas y ecuativas sin la mediación de otros elementos, ni léxicos, ni gramaticales. Presenta un sistema de determinantes (también denominados clasificadores y demostrativos) que además de su valor funcional pueden aportar información temporal, configuracional y espacial. Cuenta con un sistema de adposiciones, pseudo-aplicativos y aplicativos que aportan la información de trayectoria, lugar y orientación.

\subsection{Estructura morfológica}

\subsubsection{Nombres}

Los nombres en mocoví pueden ser raíces libres o ligadas. Las primeras pueden ocurrir por sí mismas sin ningún marcador posesivo o absolutivo, mientras que las ligadas marcan, al menos, posesión. Se distingue la posesión alienable de la inalienable según el grado de asociación entre el poseedor y el poseído. En función de esta relación, Grondona (1998) establece tres clases de nombres: Clase I (nombres que necesitan estar marcados por la posesión, aun cuando esta sea desconocida, caso en el que se marca con el prefijo $n$-); Clase II (nombres que pueden ser poseídos y que, en dicho caso, además de la marca propia de posesión, también prefijan $n$-); Clase III (nombres que nunca son poseídos).

En cuanto a la marcación de género gramatical, algunos nombres presentan género inherente. No obstante, el género se marca en la mayoría de los adjetivos, que presentan concordancia con el nombre al que especifican. Con respecto al número, los nombres pueden presentar marcas de singular (caso por defecto), paucal o plural.

Para Carrió (2009), entre los nombres simples se cuentan especialmente (i) nombre de plantas y de animales, dado que para marcar su posesión se requiere del marcador de clase 'animal' -lo, marca que soporta la posesión; y (ii) nombres relacionados con fenómenos u objetos de la naturaleza y con los momentos del día. Grondona (1998) y Gualdieri (1998) mecionan los términos que no establecen parentesco y refieren a personas.

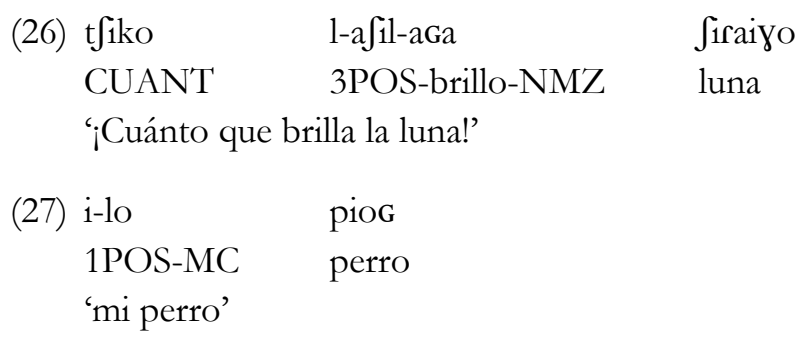


Respecto de los condicionamientos sintácticos, no se advierten diferencias en cuanto a la disposición de los nombres y las nominalizaciones.

\subsubsection{Nominalizaciones}

El mocoví dispone de diferentes sufijos para derivar nominalizaciones a partir de bases de categorías diversas. Consideramos que los sufijos reconocidos por Gualdieri (1998), constituyen dos grupos de diferente grado de productividad ${ }^{4}$. A continuación organizamos la presentación de los casos en función de las bases de derivación.

\subsubsection{Nombres denominales}

Derivar nombres a partir de bases nominales resulta ser la posibilidad menos frecuente. Para el caso del sufijo -sat, esto responde a que requiere bases altamente especificadas; deriva colectivos de base con semántica vegetal (28). Por otro lado, se distinguen dos morfemas con significado locativo, uno: -lad (29), cuya semántica es 'lugar plano y elevado'; y, -ki (30), 'lugar cerrado'. Ahora bien, en la mayoría de los casos, estos nominalizadores no se ensamblan directamente a las bases verbales sino que previamente requieren material morfológico de nominalización. Esta categorización se logra mediante el sufijo - aGa- (31).

(28) n-aka?-ni

Koyoki i-libini

ke-di

3-tirar-DIR maleta 3-poner-DIR OBL-DET

\section{akapi-sat}

'Tira la maleta, la coloca en el pastizal.'

gramilla-COL (PIO 1)

\section{(29) qa?Gan-aga-lad}

sentar-NMZ-NMZ

'asiento'

(30)

\begin{tabular}{|c|c|c|}
\hline $\begin{array}{l}\text { kaam-sa-ni } \\
\text { sentar-?-DIR }\end{array}$ & $\begin{array}{l}\text { kee-ni } \\
\text { OBL-DET }\end{array}$ & $\begin{array}{l}\text { l-omala-oki } \\
\text { 3POS-cama-DIM.M }\end{array}$ \\
\hline$v e-s a-y e \quad l a i$ & na & $-a G a-k i$ \\
\hline
\end{tabular}

EX-ASP-ALAT ADV DET mirar-NMZ-CL

'Está sentada en la camilla están al lado de la ventana.'

(PIO 5)

(31) 1-Jikia-aGa

qosot

3POS-cosquilla-NMZ

garganta

'Me pica la garganta.'

(PIO 6)

Consideramos que este afijo, $-a G a$, que es el de mayor frecuencia en esta gramática, aporta rasgos categoriales [+ nombre] pero no dispone de ningún contenido semántico asociado. En tal sentido, la gramática del mocoví parece disponer de rasgos puramente sintácticos que posibilitan la generación productiva de, en este caso, 
nombres complejos. Al respecto, esta característica se evidencia también con numerosas raíces sub-especificadas.

\subsubsection{Nombres deadjetivales}

Es posible derivar nombres a partir de bases adjetivales, de hecho el tipo semántico color no presenta formas nominales simples, mientras que sí se registran casos de formas derivadas a partir de adjetivos, esto mediante el, ya mencionado, sufijo $-a G a$.

(32)

$$
\begin{aligned}
& \text { l-tok-aGa } \\
& \text { 3POS-rojo-NMZ } \\
& \text { 'su rojo' }
\end{aligned}
$$
b. l-kola-aGa
3POS-azul-NMZ
'su azul'

Los casos de adjetivos simples parecen corresponder a los que expresan color. Por esta razón, no resulta posible determinar nombres deadjetivales correspondientes a este campo semántico. No obstante, no se descarta la posibilidad de algunos casos marginales referentes a propiedades físicas. Se cita aquí el caso de (33).

\section{(33) l-tim-aGa}

3POS-amargo-NMZ

'su amargor'

\subsubsection{Nombres deverbales}

La derivación de nombres a partir de raíces verbales es también muy frecuente y, para el caso, se logra mediante distintos agregados morfológicos.

Nuevamente atendemos al sufijo $-a G a$, que se presenta en nombres derivados de raíces verbales intransitivas. El sufijo nominalizador se adjunta, aquí, a las raíces

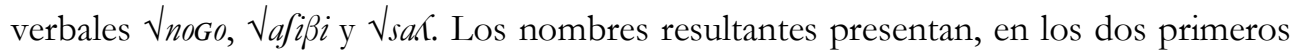
casos, el prefijo de posesión $n$ - (el cual se inserta, a nuestro juicio, ante todo contexto subespecificado). En (35), en cambio, el prefijo está especificado para la primera persona singular.

(34) a. n-noGo-aGa

IND-transpirar-NMZ

'el sudor'

(35) $i-s a k-a G a$

1POS-pesar-NMZ

'mi peso' b. $\quad n-a \int i \beta i-a G a$

IND-secar-NMZ

'la sequía'

El sufijo - $a G a$ permite expresar nombres vinculados con el evento denotado por la base verbal (se nombran los procesos: 'sudar', 'secar' y 'pesar'). En los tres casos presentados se trata de verbos de tipo intransitivo; es más, basándonos en las pruebas 
morfosintácticas de causativización morfológica, nos arriesgamos a decir, inacusativo, por lo que, en estos casos, el sufijo nominalizador se combina con raíces verbales no agentivas. ${ }^{5}$ Una prueba de ello es la selección pronominal de estas raíces, así como la selección del morfema causativizador.
a. $\boldsymbol{r}-a) i \beta i-t a$
lava
b. $n i$
r-ahasa i-asißi-Gat
$n a$

\section{la?va}
3-secar-DUR tierra
DET tierra
'La tierra está seca.'
DET sol
3A-secar-CAU
'El sol secó la tierra.'

En mocoví, para la tercera persona la prefijación de la vibrante simple indica intransitividad (36.a), mientras que la prefijación de la vocal cerrada anterior no redondeada marca agentividad plena (36.b). La morfología de causatividad requerida en (36.b) presenta a la inacusativa como forma básica. Sobre esta estructura argumental se deriva el nominal.

A la alta frecuencia de $-a G a$, se suman 4 morfemas que permiten generar nombres con diferencias semántica bien marcadas: -aGak, para nombres eventivos; -ek, para nombres resultativos; - - $a$ t, para nombres instrumentales; y, $-a G$, para nombres agentivos.

El afijo -aGak (37) es un sufijo nominalizador eventivo (Carrió, 2009) que deriva formas equivalentes a los infinitivos de las lenguas románicas, pero con marcas pronominales de posesión correferentes con el sujeto del verbo base del que deriva (ver Introducción). Cabe considerar aquí que las raíces verbales no pueden aparecer libres en mocoví, requieren obligatoriamente de, al menos, la marca de persona gramatical.

(37) na leviye i-ve i-asot-ta-agak

DET música 3-EX 1POS-bailar-DUR-NMZ

'La música me hace bailar. (Lit. La música causa mi bailar.)'

En (37) la nominalización satura una posición argumental habilitada por la construcción causativa factitiva. El existencial (ve), verbo léxico especificado, lexicaliza la causa para promover un argumento externo, a la vez que se ensambla con el nombre deverbal eventivo que satura la posición de argumento interno en el que la marca de posesión recupera el sujeto causado (primera persona, en este caso).

En cuanto al morfema -ek, éste permite la generación de nombres que hacen referencia a un resultado u objeto típico de acción y, al igual que en el caso de los nombres eventivos, presentan marcación morfológica de posesión correferencial con el sujeto del verbo base, tal como muestra la gramaticalidad de (38) y la agramaticalidad de (39). 
(38) xuan $i_{\mathrm{i}}$-okatfaa

Juan 3-robar so $\quad i_{\mathrm{j}}$-owen-ek

DET 1POS-trabajar-NMZ

'Juan robó mi trabajo.'

(39) * maria $r_{\mathrm{i}}-e \beta o s e$

na $\quad i_{\mathrm{j}}$-eßol-ek

María 3-cocinar

DET 1POS-cocinar-NMZ

'María cocina mi comida. (Lit.: María cocina mi comida cocinada por mí.)'

Las construcciones resultativas en esta lengua devienen nominalizaciones de objeto. Por otro lado, la nominalización de instrumental mediante -Gat se logra a partir de una raíz verbal transitiva antipasivizada (40), complejo que hospeda la materialización de los rasgos nominales.

(40) a.

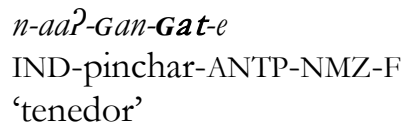

b. l-kijo-Gan-Gat

3POS -lavar-ANTP-NMZ.M

'su jabón (de él/ella)

La marca de nominalización instrumental es isomórfica a la marcación de causativa morfológica. Esto no es extraño en las lenguas del mundo, un instrumento que causa un evento se puede materializar en la sintaxis mediante un sintagma oblicuo y mediante un sujeto (Levin, 1993). Para este último caso se requiere de nominalizaciones instrumentales como las de (40). Esto es, nominalizaciones capaces de saturar posiciones argumentales y de insertarse en posiciones nucleares en el dominio nominal. Nótese que para la buena formación de estos casos, se requiere de la prefijación de marcas de posesión que, a diferencia de lo acontecido con los nominales resultativos y eventivos, no involucran rasgos [+ agentivos].

Por último, entonces, entre los nombres deverbales se encuentra también el caso del nominalizador agentivo, el cual, valiéndose del afijo - $a G$ (41), deriva nombres que hacen referencia al agente de la acción expresada en el verbo.

(41) n-emeen-a-Gan-aG

IND-vender-VE-ANTP-NMZ.M

'vendedor'

Las nominalizaciones agentivas presentan la misma estructura que las de instrumento. Se ensamblan a bases verbales transitivas, luego reciben rasgos de antipasiva, reduciendo así la estructura argumental de la raíz, para por último hospedar morfología nominal agentiva. La prefijación de posesión indefinida, resulta ser una operación constante en las nominalizaciones. Con una mínima excepción de nombres simples, semánticamente restringidos, el resto de los nombres y nominalizaciones de la lengua presentan marcas de posesión con diferentes rasgos asociados. 


\subsection{Naturaleza categorial}

A fin de determinar el estatuto categorial de las formas disponibles en esta gramática particular, cabe describir sucintamente las particularidades morfológicas de los nombres en relación con los adjetivos y los verbos. En esta lengua los nombres presentan comportamientos morfológicos y sintácticos que los acercan a los adjetivos y a los verbos. Sin embargo, también se reconocen diferentes características que los distinguen. A continuación se detallan las semejanzas y las diferencias más relevantes.

\subsubsection{Nombres o adjetivos}

Ambas categorías pueden constituirse como palabras monomorfemáticas (42); y, pueden alojar sufijos flexivos isomórficos para codificar género y número (43)-(44). Se reconoce para ambas categorías el sufijo de género masculino $-k$, el sufijo de paucal masculino -qa y el sufijo de plural -ipi.
(42) a. rabasa
sol'
b. tok
'rojo'
(43) a. l-iale-k
b. liale-qa
3POS-hijo-PC.M
'sus hijos'
3POS-hijo-M
c. liale-qa-ipi
3POS-hijo-PC.M-PL
'sus hijos'
(44) a. l-aya-aGa-ai-k
3POS-feo-NMZ-M
'feo'
b. l-aya-aGa-qa
3POS-feo-NMZ-PC.M 'feos'
c. l-aya-aGa-qa-ipi
3POS-feo-NMZ-PC.M-PL
'feos'

Las dos categorías pueden hospedar un morfema de diminutivo, frecuente, semántico. amalgamado con marca de género (45) y con el mismo valor

(45) a. pioq-oki perro-DIM.M
'perrito' b. l-aya-aGa-ai-k-oki

3POS-feo-NMZ-ADJ-M-DIM.M

'feito'

Las dos categorías pueden hospedar un morfema de diminutivo, frecuente, amalgamado con marca de género (45) y con el mismo valor semántico.
(45) a. pioq-oki
b. l-aya-aGa-ai-k-oki
perro-DIM.M
'perrito'
3POS-feo-NMZ-ADJ-M-DIM.M
'feito'

Asimismo, ambas pueden funcionar como predicados primarios (46), en dicho caso denotan propiedades de las entidades a las que modifican:
(46) a. qonok
POS.comida 3POS-salado-NMZ
'Nuestra comida está salada y amarga.'

\section{l-tim-aGa}
3POS-amargo-NMZ


b. ni i-atee naGan-aGa-rai

DET 1POS-madre bueno-NMZ-ADJ.F

'Mi madre es buena.' $\quad$ (PIO 2)

Si se advierte que en la gramática de esta lengua no se dispone de formas verbales copulativas que permitan generar estructuras predicativas primarias, entonces, es importante considerar que las construcciones del tipo $[\mathrm{N}+\mathrm{N}],[\mathrm{N}+$ CUANT $+\mathrm{N}]$ y $[\mathrm{N}$ + A] implican necesariamente una función predicativa respecto del nombre al que acompañan. Así, en (46.a) los nombres litißiaGa ('salada') y litimaGa ('amarga') predican sobre el nombre qonok ('comida'). A su vez, en (46.b), el adjetivo naGanaGarai ('buena') predica sobre el nombre iatee ('mi.madre').

Ahora bien, hemos puesto de manifiesto las características que acercan estas dos formas categoriales. No obstante esto, ambas categorías presentan diferencias en cuanto a los tipos semánticos que denotan y a sus comportamientos morfológicos y sintácticos. A continuación, se recuperan estas distinciones, análisis que permitirá mostrar que el nombre es una categoría que, a diferencia del adjetivo, reviste mayor frecuencia en esta lengua.

Si se atiende a la semántica de las construcciones, y siguiendo a Baker (2004), el nombre es la única clase de palabra que puede designar a una misma entidad con referencia constante, en este sentido, es la única categoría que posee criterio de identidad. Sólo los nombres pueden constituirse en expresiones referenciales. Por lo tanto, el nombre, a diferencia del adjetivo, puede ocupar posiciones argumentales (ver casos de 3.1.2).

En mocoví, es posible reconocer que el nombre es la categoría que, por un lado, denota entidades tales como animales, objetos, elementos de la naturaleza, etc., cuya referencia puede perdurar a lo largo del tiempo (es decir, suponen referencia tanto constante como variable) (47); mientras que además tiene la posibilidad de denotar algunos de los tipos semánticos tradicionalmente asociados, en otras lenguas, a los adjetivos (Dixon, 1982) (48).

(47) rahasa komak-ai-k

sol grande-ADJ-M

'El sol es grande.'

(48) a. iqolaga ('su azul')

color

b. koGo ('vejez')

edad

c. naGanaGa ('bondad')

valor

d. letisaga ('renguera')

propensión física

e. netonaga ('alegría')

propensión humana

Si bien pueden reconocerse adjetivos que denotan estos conceptos en la lengua, el nombre resulta una clase muy frecuente con la cual pueden expresarse estos tipos 
semánticos. Así, para expresar propiedades transitorias una estrategia muy productiva en la lengua es recurrir a construcciones existenciales del tipo $[\mathrm{n}+\mathrm{ex}+\mathrm{n}]$ :

(49) xuan ve l-sile

Juan EX POS-suciedad

'Juan está sucio.'

En este caso el existencial permite expresar una relación de posesión entre un sintagma poseedor ('Juan') y uno poseído ('su.suciedad'). Este tipo de estructura permite expresar estados transitorios. En contraste, las formas adjetivas en posición predicativa habilitan lecturas vinculadas con propiedades inherentes de las entidades a las que modifican:

(50) jim Jile-aGa-ai-k

PRO1 suciedad-ADJ-M

'Yo soy sucio.'

Las construcciones del tipo $[\mathrm{n}+\mathrm{ex}+\mathrm{n}]$ son seleccionadas por los hablantes para expresar propiedades transitorias mientras que las construcciones [n+adj] permiten manifestar propiedades inherentes de los individuos sobre los que predican.

En cuanto a la codificación numérica, esta puede manifestarse morfológicamente sólo en el nombre, sólo en el determinante o bien en ambos elementos. En cambio el adjetivo puede, o no, flexionar en número para concordar con el nombre al que modifica. Sin embargo, en ningún caso puede ser el único elemento dentro del sintagma que codifique la información numérica. Así, el nombre se diferencia del adjetivo además, por poder ser el único elemento que soporta las marcas flexivas de número dentro del sintagma determinante.

Las formas adjetivas pueden ser recategorizadas en nombres si son seleccionadas por determinantes, lo cual les permite ocupar posiciones argumentales (51). En cambio, no pueden sufrir este tipo de recategorización si aparecen en sintagmas escuetos.
(51) a. marina
O-koßi-ta-Gat
Marina
3-gustar-DUR-CAU
na tok
DET rojo
'A Marina le gusta el rojo.'
b. ${ }^{*}$ na
DET perro EX
'El perro existe negro.'
l- $\beta$ er-aGa-ai-k
3-negro-NMZ-ADJ-M

En este sentido, y siguiendo a Baker (2004), solo los sintagmas nominales pueden aparecer con determinantes que marquen distinciones del tipo definido-indefinido. Por este motivo, el contexto de determinante coerciona la conversión de la categoría adjetivo en un nombre. 


\subsubsection{Nombres o verbos}

Atendiendo ahora a la delimitación entre nombres y verbos, conviene citar a Croft $(1990,2001)$ quien sostiene que las combinaciones prototípicas o no marcadas para nombre, adjetivo y verbo son la referencia, la modificación y la predicación respectivamente, estableciendo correspondencias entre las categorías ontológicas y las categorías léxicas. Por otro lado, Langacker (1987) considera que todas las categorías gramaticales deben definirse semánticamente independientemente de las propiedades formales particulares con que se materialicen en las lenguas; luego, podrán aplicarse a casos no prototípicos. En esta línea de análisis, para Croft (2000) nombre y verbo no son categorías de las lenguas particulares, sino universales lingüísticos dado que constituyen prototipos tipológicos. Haspelmath (2010), por su parte, propone atender a criterios formales específicos para determinar las categorías descriptivas de las lenguas. En tal sentido será necesario buscar las propiedades estructurales específicas de cada lengua y, a la vez, los conceptos que permiten compararlas para dar lugar a prototipos. En esta puesta en comparación se esperan tendencias prototípicas, por ejemplo, para Croft es de esperar que las palabras que designan objetos concretos sean nombres prototípicos y las palabras que designan acciones sean verbos (principio de categorialidad), para Givón (1984) la denotación prototípica de nombres, adjetivos y verbos está determinada por una escala de estabilidad temporal (de menor a mayor para estos casos). Ahora bien, claro está que esta base ontológica no puede servir por sí sola para la categorización léxica, los límites de estas relaciones son lábiles y esto es lo permite que conceptos similares presenten categorizaciones diferentes en las distintas lenguas.

Ahora bien, atendemos aquí el caso del mocoví. Mientras que los nombres, tal como se mostró, constituyen palabras simples, los verbos se materializan siempre con estructura interna compleja. En todos los casos, los verbos están marcados con, al menos, información pronominal. Sumado a ello, nombres y verbos en mocoví alojan morfología de persona y de número; los verbos no marcan género; y, hasta donde sabemos, ninguno de los dos casos marca gramaticalmente el tiempo. Sí alojan morfología de diminutivo, combinación altamente frecuente para el caso de los nombres, y muy restringida para el caso de los verbos ${ }^{6}$.

Anteriormente se describieron las nominalizaciones de objeto afectado (nominalizaciones resultativas) en las que la marca de posesión prefijada a las nominalizaciones indica el sujeto agentivo de la construcción, razón por la cual, por ejemplo, las construcciones argumentales con rasgos [- animado] constituyen una restricción para su combinación. Esta marca es la única indicación del sujeto en las construcciones resultativas.

En cuanto a las similitudes formales, también se observan ciertas nominalizaciones deverbales (no analizadas más arriba) que aparecen, en las oraciones, como predicado 
principal. Es el caso de las nominalizaciones de sujeto afectado, fenómeno muy poco frecuente. En estas construcciones de sujeto afectado, el sujeto agente se expresa mediante un sintagma oblicuo.

(52) $i$-aman-ek na nai

1 -gustar-NMZ DET pescado

'Me gusta (comer) pescado.'

(53) ni l-amaGaski i-aval-ek

DET 3POS-camisa 1-prestar-NMZ OBL Juan

'Esta camisa me prestó Juan.'

Ahora bien, en relación al contexto de distribución sintáctica, las nominalizaciones de objeto afectado (resultativas), siempre serán núcleo de un sintagma nominal y nunca aparecerán como predicado principal en una oración simple. Aquí, el nombre indicará el producto de la acción expresada por la base verbal transitiva, a la vez que el sujeto original está presente en la flexión de persona. A diferencia de ello, en las nominalizaciones de sujeto afectado, marginales en esta gramática y también derivadas de bases verbales eventivas, el sujeto se expresa mediante las marcas de genitivo, pero aquí, no se constituye un sintagma nominal canónico sino que el verbo base de la nominalización deverbal cumple el papel de verbo principal en la oración. Ahora bien, dichas marcas de genitivo, evidencia de la herencia argumental en las nominalizaciones resultativas, no pueden ser interpretadas en estos casos de sujeto afectado como con rasgo [+ agentivo]. La poco prolífera aparición de la construcción dificulta el trabajo en campo, lo que impacta en la elucidación del fenómeno.

Por último entonces, las formas derivadas mediante el afijo - $a G a$ resultan de alta frecuencia también en la combinación con el verbo existencial para generar predicaciones de tipo secundarias. Estas asociaciones conllevan un alto valor predicativo y, en ellas, el verbo existencial parece funcionar más como una marca lexicalizada para habilitar la predicación, que como un verbo pleno.

$\begin{array}{lll}\text { xuan ve l-tok-aGa } & \text { l-afik } \\ \text { Juan EX } & \text { 3POS-rojo-NMZ } & \text { 3POS-rostro } \\ \text { 'Juan se ruborizó. (Lit.: Juan hay rojo de su rostro.)' }\end{array}$

Aquí, el nominal letuaga ('su rojo') se genera sobre la raíz adjetiva tok, a la que se ensambla el nominalizador $-a G a$; esto habilita la prefijación al nominal, de la marca de genitivo de tercera persona. Es esta construcción, entonces, la que, junto al verbo existencial como soporte, permite lograr la semántica incoativa.

\section{CONCLUSIONES}

En este trabajo, se propuso un análisis de la categoría 'nombre' en la gramática del mocoví teniendo en cuenta las particularidades morfológicas y sintácticas que presenta en la lengua. En función de este objetivo, se describió la organización de su gramática, 
la operatividad de los rasgos disponibles para generar nominalizaciones y las restricciones morfológicas y sintácticas que manifiestan los nombres, y que los diferencian de los adjetivos y los verbos de la lengua. En cuanto a los rasgos disponibles en el inventario de la gramática, se pudo reconocer que el mocoví parece disponer de rasgos puramente sintácticos que posibilitan la generación productiva de nombres complejos, como ser, aquellos derivados por el sufijo - $a G a^{-}$. A su vez, se identificaron rasgos semánticos asociados a diferentes sufijos nominalizadores, tales como: locación, semántica vegetal, eventividad, resultado, instrumento y agentividad.

Con respecto a la generación de nominalizaciones, se reconocieron pocos casos de nombres derivados de adjetivos, los cuales se generan principalmente a partir de raíces adjetivas que expresan color. A su vez, se reconocieron diferentes sufijos que derivan nombres a partir de verbos. En cada caso, se delimitaron las especificaciones semánticas de cada sufijo, las cuales condicionan sus contextos de inserción, esto es, determinan las bases con las cuales pueden combinarse.

En cuanto a la delimitación categorial del nombre dentro de la gramática, si bien hay zonas difusas entre los fenómenos analizados, la evidencia morfológica y sintáctica a favor de la diferenciación de la categoría nominal en relación con la verbal y adjetival, resulta contundente. $\mathrm{Al}$ respecto, en este trabajo se presentaron las semejanzas y diferencias entre el nombre y el adjetivo, por un lado y entre el nombre y el verbo, por el otro. En cuanto a las semejanzas entre el nombre y el adjetivo, se reconoció que ambas categorías pueden constituirse como palabras monomorfemáticas. Asimismo, pueden alojar sufijos flexivos isomórficos para codificar género y número. Las dos categorías pueden hospedar un morfema de diminutivo amalgamado con marca de género y con el mismo valor semántico. A su vez, ambas pueden funcionar como predicados primarios, en dicho caso denotan propiedades de las entidades a las que modifican. Se reconoció también que ambas categorías pueden codificar los tipos semánticos tradicionalmente asociados con los adjetivos (Dixon, 1982). Sin embargo, en este punto, se especificó que el nombre, a diferencia del adjetivo, expresa entidades cuya referencia perdura a lo largo del tiempo. En cuanto a las diferencias, se observó que sólo los nombres pueden ocupar posiciones argumentales. Sumado a esto, permiten expresar estados transitorios mientras que los adjetivos expresan propiedades inherentes de las entidades a las que modifican. Finalmente, se señaló que los nombres pueden ser los únicos elementos dentro del sintagma determinante que codifiquen información numérica, mientras que los adjetivos no presentan esa posibilidad.

En relación al nombre y al verbo, se observó que ambos pueden alojar morfología de persona y de número, pero sólo los nombres pueden marcar género. Hasta donde sabemos, ninguno de los dos casos marca gramaticalmente el tiempo. Otro aspecto que comparten es la posibilidad de alojar la marca de diminutivo, si bien con mucha mayor restricción para el caso de los verbos. Sumado a esto, se observó que mientras 
que los nombres pueden ser simples, los verbos siempre presentan una estructura compleja. Asimismo, se reconoció que tanto el verbo como el nombre participan de construcciones predicativas. Se reconocieron dos casos para el nombre: en primer lugar, las nominalizaciones de sujeto afectado, en las cuales los nombres, al igual que los verbos, pueden funcionar como predicados principales en una oración simple; en segundo lugar, la construcción [ex $+n$ ] es una estructura productiva que habilita predicación secundaria, y con la cual pueden lograrse predicaciones con semántica incoativa. Finalmente, se marcó y reconoció, para el caso del mocoví, raíces no categorizadas.

\section{REFERENCIAS BIBLIOGRÁFICAS}

Arad, M. (2003). Locality constraints on the interpretation of roots: The case of Hebrew denominal verbs. Natural Language and Linguistic Theory, 21, 737- 778.

Baker, M. (2004). Adjectives as neither nouns nor verbs. En M. Baker (Ed.), Lexical categories: Verbs, Nouns, and Adjectives (pp. 190-263). Cambridge, UK: Cambridge University Press.

Bobaljik, J. (2015). Distributed morphology. Ms. Capitulo de Encyclopaedia [en línea]. Disponible en: http://bobaljik.uconn.edu/papers/DM_ORE.pdf

Bucca, S. (1979). Palabras y frases mocovies de Colonia Dolores recopiladas en Colonia Dolores. Gobernador Crespo. Santa Fe. Editada luego como: Palabras y frases mocovíes de Colonia Dolores (1981). Cuadernos del Sur, 14, 231-238.

Buckwalter, A. S. \& Buckwalter, L. L. (Recop.). (2004). Vocabulario Castellano- Guaycurú. Formosa, Argentina: Equipo Menonita.

Buckwalter, A. S. \& Ruiz, R. (2000). Mocoví. South American Indian Languages, Computer Database (Intercontinental Dictionary Series, vol. 1). General Editor Mary Ritchie Key. Irvine: University of California, CD-ROM.

Carrió, C. (2009). Mirada generativa a la Lengua Mocoví (Familia Guaycurú). Tesis Doctoral, Universidad Nacional de Córdoba, Córdoba, Argentina.

Carrió, C. (2015a). Alternancias verbales en Mocoví (familia guaycurú, Argentina). Revista Lingüística, 31(2), 9-26.

Carrió, C. (2015b). Construcciones causativas y anticausativas en Mocoví. Revista LIAMES - Linguas Indigenas Americanas, 15(1), 69-89.

Croft, W. (1990). Typology and universals. Nueva York: Cambridge University Press

Croft, W. (1991). Syntactic categories and grammatical relations: The cognitive organization of information. Chicago: University of Chicago Press. 
Croft, W. (2000). Parts of speech as language universals and as language-particular categories. En P. M. Vogel \& B. Comrie (Eds.), Approaches to the typology of word classes (pp. 65-102). Berlín / Nueva York: de Gruyter Mouton.

Croft, W. (2001). Radical construction grammar: Syntactic theory in typological perspective. Oxford: Oxford University Press.

Dixon, R. M. W. (1982). Where have all the adjectives gone? En R. M. W. Dixon (Ed.), Where have all the adjectives gone? And other essays in Semantic and Syntax (pp. 1-62). Berlín: De Gruyter Mouton.

Embick, D. (2015). The morpheme. A theoretical introduction. Berlín: De Gruyter Mouton.

Embick, D. \& R. Noyer (2004). Distributed morphology and the syntax/morphology Interface. En G. Ramchand \& C. Reiss (Eds.), The Oxford Handbook of Linguistic Interfaces (pp. 289-324). Oxford: Oxford University Press.

Givón, T. (1984). Syntax: A functional-typological introduction. Ámsterdam: John Benjamins.

Grondona, V. M. (1998). A grammar of mocovi. Tesis doctoral, University of Pittsburgh, Pittsburgh, Estados Unidos.

Gualdieri, B. (1998). Mocovi (Guaycuru) Fonologia e morfossintaxe. Tesis doctoral, Universidade Estadual de Campinas, Campinas, Brasil.

Gualdieri, B. (2003). La palatalización en mocoví. Informe presentado para el proyecto Endangered Languagues - Endangered Peoples of Argentina. Buenos Aires: Facultad de Filosofía y Letras, Universidad de Buenos Aires - Dept. of Linguistics, Max Planck Institute [en línea]. Disponible en: http://dobes.caicyt.gov.ar/corpora/dobes/Chaco/Mocovi/Studies/Linguisti cs/Phonology/Annotations/clmocovifono2.pdf

Halle, K. \& Marantz, A. (1993). Distributed morphology and the pieces of inflection. En K. Hale \& S. Keyser (Eds.), The View from Building 20 (pp. 111-176). Cambridge: MIT Press.

Harley, H. (2009). The morphology of nominalizations and the syntax of vP. En M. Rathert \& A. Giannankidou (Eds.), Quantification, Definiteness and Nominalization (pp. 320-342). Oxford: Oxford University Press,

Harley, H. (2014). On the identity of roots. Theoretical Linguistics, 40(3-4), 225-276. DOI: https://doi.org/10.1515/tl-2014-0010. Berlín: De Gruyter Mouton.

Haspelmath, M. (2010). Comparative concepts and descriptive categories in crosslinguistic studies. Language, 86(3), 663-687. 
Jara, V. (en desarrollo). Nominalizaciones deverbales en mocoví. Tesina de Licenciatura, Universidad Nacional del Litoral, Santa Fe, Argentina.

Juárez, C. (2013). Sistemas de alineación en el mocoví (guaycurú) hablado en Colonia Aborigen (Chaco, Argentina). Tesis de magíster, Universidad de Sonora, Sonora, México.

Langacker, R. (1987). Nouns and verbs. Language, 63(1), 53-94.

Levin, B. (1993). English verb classes and alternations. Chicago: University of Chicago Press.

Marantz, A. (1997). No escape from syntax: Don't try morphological analysis in the privacy of your own lexicon. En A. Dimitriadis, L. Siegel \& C. Surek-Clark (Eds.), Proceedings of the 21st Penn Linguistics Colloquium (pp. 201-225). Philadelphia: UPenn Working Papers in Linguistics.

Marantz, A. (2001). Words. Ponencia presentada en el 20th West Coast Conference on Formal Linguistics. University of Southern California, California, USA.

Rabasedas, M. I. (2016). La marcación de concordancia morfológica del verbo con el sujeto y con el objeto en mocoví. Tesina de Licenciatura, Universidad Nacional del Litoral, Santa $\mathrm{Fe}$, Argentina.

\section{ABREVIATURAS}

1: primera persona; 3: tercera persona; A: agente; $\mathrm{ADJ}$ : adjetivo; $\mathrm{ADV}$ : adverbio; $\mathrm{AF}$ : afectado; AG: agentivo; ALAT: alativo; ANTP: antipasiva; APL: aplicativo; ASP: aspecto; CAU: causativo; CL: clasificador; COL: colectivo; COM: comitativo; CONJ: conjunción; CUANT: cuantificador; DET: determinante; DIM: diminutivo; DIR: direccional; DUR: durativo; EV: eventivo; EX: existencial; F: femenino; IND: indefinido; INSTR: instrumental; M: masculino; MC: marcador de clase; NMZ: nominalización; OBL: oblicuo; PC: paucal; PL: plural; POS: posesivo; PRO: pronombre; PROS: progresivo; PROG: prospectivo; RES: resultativo; SG: singular; VE: vocal epentética.

\section{NOTAS}

1 Agradecemos a los referentes-consultores de la lengua mocoví: Alfredo Salteño, Delfino Nacitiquí, Raúl Teotí, Cristina Vázquez, Rufino Vázquez y Hugo Balcarse. Todos los errores e inconsistencias son de nuestra responsabilidad. El trabajo de campo se financió parcialmente con aportes del Proyecto de Investigación Orientado ASACTEI Ministerio de Ciencia, Tecnología e Innovación-Provincia de Santa Fe (2010-031-16). 
2 Reconocemos que no puede, en principio, asumirse una única variedad chaqueña. No obstante, a fin de simplificar la exposición, nos referimos con este término a las posibles variedades (en estudio) de la lengua hablada en la región chaqueña.

${ }^{3}$ Fuente: PIO, i.e.: corpus de textos recopilados en el marco del Proyecto de Investigación Orientado en Red en Ciencias Sociales "Creación de recursos informáticos para las lenguas minoritarias Mocoví y Quechua” (Ministerio de Ciencia y Tecnología del Gobierno de la Provincia de Córdoba).

${ }^{4}$ En este análisis, no se consideran como morfemas nominales los casos -le/-lek; -se/ -sek, reconocidos por Gualdieri (1998).

5 Para profundizar respecto de la relación causativización morfológica diferenciada para inacusativos en mocoví (Carrió, 2015b).

${ }^{6}$ Las razones que restringen la presencia de la marca de diminutivo en las bases verbales son aún desconocidas, si bien probablemente se vinculen con la marcación aspectual de ciertos verbos eventivos. 